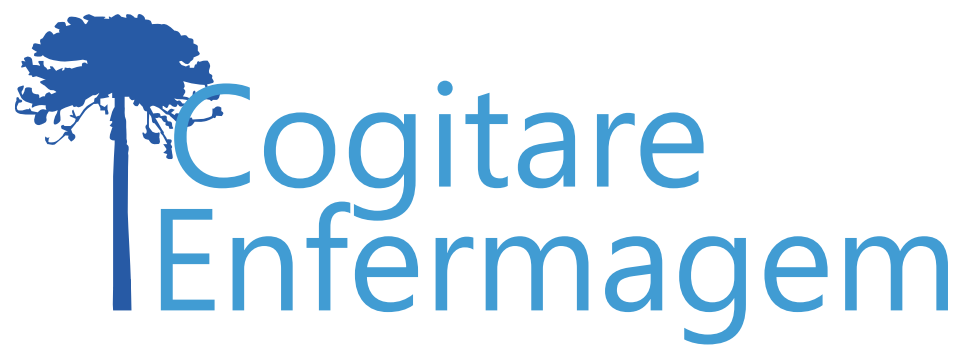

\title{
CUIDADOS DE ENFERMAGEM NO PROCESSO DE EXTUBAÇÃO OROTRAQUEAL: REVISÃO INTEGRATIVA
}

Tessy Nnonyelum Miozzo Ezeagu1, Anna Carolina Gaspar Ribeiro²

\section{RESUMO}

Objetivo: descrever quais os cuidados de enfermagem no processo de extubação orotraqueal de pacientes em Unidade de Terapia Intensiva.

Método: revisão integrativa com busca de dados entre junho de 2017 e fevereiro de 2018 nas seguintes bases: Medline/PubMed, Literatura Latino-Americana e do Caribe em Ciências da Saúde, Web of Science, Base referencial da Editora Elsevier, Banco de Dados em Enfermagem, Índice Bibliográfico Espanhol de Ciências da Saúde, e Portal da Agência Nacional de Vigilância Sanitária do Ministério da Saúde.

Resultados: nos 12 artigos eleitos por estarem em conformidade com os critérios de inclusão, foram identificados 16 cuidados de enfermagem que se resumem em três momentos: antes, durante e depois da extubação propriamente dita.

Conclusão: no processo de extubação orotraqueal, a aplicação de cuidados de enfermagem baseada em evidências é essencial para assegurar a segurança do paciente, prevenindo complicações, reduzindo tempo de internação e custos.

DESCRITORES: Enfermagem; Extubação; Segurança do Paciente; Unidades de Terapia Intensiva; Educação Continuada.

COMO REFERENCIAR ESTE ARTIGO:

Ezeagu TNM, Ribeiro ACG. Cuidados de enfermagem no processo de extubação orotraqueal: revisão integrativa. Cogitare enferm. [Internet]. 2019 [acesso em "colocar data de acesso, dia, mês abreviado e ano"]; 24. Disponível em: http://dx.doi.org/10.5380/ce.v24i0.58144.

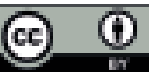

Este obra está licenciado com uma Licença Creative Commons Atribuição 4.0 Internacional. 


\title{
NURSING CARE IN THE OROTRACHEAL EXTUBATION PROCESS: INTEGRATIVE REVIEW
}

\begin{abstract}
Objective: To describe the nursing care in the process of orotracheal extubation of patients in an Intensive Care Unit.

Method: Integrative review with data collected between June 2017 and February 2018 through search to the following databases: Medline/PubMed, Literatura Latino-Americana e do Caribe em Ciências da Saúde (LILACS), Web of Science, Elsevier's Scopus Database, Banco de dados em enfermagem (BDENF), IBECS (Spanish Bibliographical Index in Health Sciences), and Portal of ANVISA (National Health Surveillance Agency of the Ministry of Health).

Results: In the 12 articles selected for meeting the inclusion criteria, 16 types of nursing care were identified, which are summarized in three moments: before, during and after extubation. Conclusion: In the process of orotracheal extubation, the use of evidence-based nursing care is essential to ensure patient safety, prevent complications, and reduce hospitalization time and costs.
\end{abstract}

DESCRIPTORS: Nursing; Extubation; Patient safety; Intensive Care Units; Continuing Education.

\section{CUIDADOS DE ENFERMERÍA EN EL PROCESO DE EXTUBACIÓN OROTRAQUEAL: REVISIÓN INTEGRAL}

\section{RESUMEN}

Objetivo: describir los cuidados de enfermería en el proceso de extubación orotraqueal de pacientes en Unidad de Terapia Intensiva.

Método: revisión integral con búsqueda de datos hecha entre junio de 2017 y febrero de 2018 en las siguientes bases: Medline/PubMed, Literatura Latinoamericana y del Caribe en Ciencias de la Salud, Web of Science, Base referencial del Editorial Elsevier, Banco de Datos en Enfermería, Índice Bibliográfico Español de Ciencias de la Salud, y Portal de la Agencia Nacional de Vigilancia Sanitaria del Ministerio de la Salud.

Resultados: en los 12 artículos seleccionados de acuerdo a la conformidad con los criterios de inclusión, se identificaron 16 cuidados de enfermería, lo cuales se resumen en tres momentos: antes, durante y después de la dicha extubación.

Conclusión: en el proceso de extubación orotraqueal, la aplicación de cuidados de enfermería basada en evidencias es esencial para garantizar la seguridad del paciente, previniendo complicaciones, reduciendo tiempo de ingreso y costos.

DESCRIPTORES: Enfermería; Extubación; Seguridad del Paciente; Unidades de Terapia Intensiva; Educación Continuada. 
Observa-se que as práticas de enfermagem geram redução significativa na incidência de complicações associadas as intervenções de saúde, como as infecções relacionadas a assistência de saúde (IRAS), que são associadas ao tubo orotraqueal (TOT) utilizado para ventilação mecânica ${ }^{(1-2)}$. Por isso, a educação continuada da equipe de enfermagem é uma das práticas que pode ser um mecanismo propulsor de mudança de condutas, que assegurem a atenção à saúde baseada nas melhores evidências ${ }^{(3)}$.

Desta forma, o enfermeiro deve planejar e organizar intervenções de forma sistematizada e promover práticas de enfermagem baseadas em conhecimentos científicos, a fim de promover a assistência de enfermagem qualificada, visando evitar ou reduzir os riscos de tais complicações, pois contribuem para o aumento de morbidades, tempo de permanência no leito, recuperação tardia, custos hospitalares e mortalidade dos pacientes em Unidade de Terapia Intensiva (UTI) ${ }^{(4-7)}$.

Nestes casos, o conhecimento dos cuidados com paciente com TOT é fundamental para prevenção de pneumonia associada à ventilação (PAV), uma vez que esta condição é facilitada pela colonização bacteriana causada pela broncoaspiração de secreções contaminadas. Outras complicações que podem ser causadas pela presença do TOT são: lesão de laringe e traqueia, ocasionadas por injúria e ulceração decorrente da insuflação excessiva do cuff, e extubação acidental prematura, a qual pode ser causada por agitação, depressão e ansiedade do paciente, levando a reintubação e consequentemente exposição prolongada ao $\mathrm{TOT}^{(8)}$.

Dessa forma, os cuidados de enfermagem em UTI para retirada do TOT, ou seja, durante o processo de extubação orotraqueal, devem ser embasados nas evidências cientificas mais atuais a fim de padronizá-los dentro da rotina da UTI $(9,10)$. Questionamos então: quais são os cuidados de enfermagem efetivos para o processo de extubação orotraqueal segura do paciente em UTI?

Durante a prática como residente de enfermagem em uma UTI cardíaca, percebeuse que a ausência de uma rotina para esses cuidados limitou o planejamento correto de intervenções. A ausência de uma rotina de cuidados de enfermagem que indique quais são os cuidados principais durante o processo de extubação orotraqueal do paciente em estado crítico, remete a uma recuperação mais lenta, além de outras complicações associadas.

O objetivo desta revisão foi descrever quais são os cuidados de enfermagem para o processo de extubação de pacientes de uma UTI.

\section{MÉTODO}

Optou-se por realizar uma revisão integrativa, cujo método busca a síntese de dados encontrados em pesquisas de um determinado tema, que fornece informações sobre um assunto ou problema para gerar um conjunto de conhecimentos sobre o mesmo, incluindo a análise de pesquisas relevantes para proporcionar uma visão completa desse tema ${ }^{(11)}$.

Para construção da revisão, foram percorridas etapas de acordo com o referencial metodológico de Ganong: identificação do tema e seleção de uma questão norteadora; estabelecimento de critérios para inclusão e exclusão de estudos e definição da população na literatura; definição das informações dos estudos elegidos e sua avaliação; interpretação dos dados, discussão da literatura e síntese dos conhecimentos encontrados ${ }^{(12)}$.

Para a construção da questão norteadora deste trabalho, utilizou-se a estratégia PICO: P - população e problema; I - intervenção; C - comparação e O - outcome (desfecho). Assim, considerou-se P: pacientes adủltos em unidade de terapia intensiva; I: 
cuidados de enfermagem no momento da extubação endotraqueal; C: não se aplica, uma vez que não se buscou a comparação entre os cuidados; O: prevenção de complicações associadas a extubação(12). Neste sentido, a pergunta elaborada foi: Quais são os cuidados de enfermagem no momento da extubação do paciente em Unidade de Terapia Intensiva?.

A busca na literatura foi realizada entre os meses de junho de 2017 a fevereiro de 2018 nas seguintes bases de dados: The National Library of Medicine (NLM) em Medline/ PubMed, Literatura Latino-Americana e do Caribe em Ciências da Saúde (LILACS), Web of Science, Base referencial da Editora Elsevier (SCOPUS), Banco de dados em enfermagem (BDENF), Índice Bibliográfico Espanhol de Ciências da Saúde (IBECS) e no Portal da Agência Nacional de Vigilância Sanitária (ANVISA) do Ministério da Saúde do Brasil. Utilizou-se os descritores "enfermagem" e "extubação" bem como seus correspondentes nas línguas inglesa e espanhola, identificados em Descritores em Ciências da Saúde (DeCS) e Medical Subject Headings (MeSH), associados pelo operador booleano AND. Além disso, foi realizada uma busca manual nas referências citadas para confirmar se o título e o resumo dos estudos encontrados estavam em conformidade com o tema do presente estudo.

O recorte temporal envolveu estudos publicados entre os anos de 2012 e 2018, disponibilizados na íntegra, nos idiomas inglês, português e espanhol, que incluíam uma população ou amostra de pacientes maiores de 18 anos internados em UTIs e que tivessem título e resumo relacionados ao tema deste trabalho. Foram excluídos os estudos científicos de revisões de literatura e estudos de caso. A Figura 1 elucida a pesquisa em bases de dados realizada.

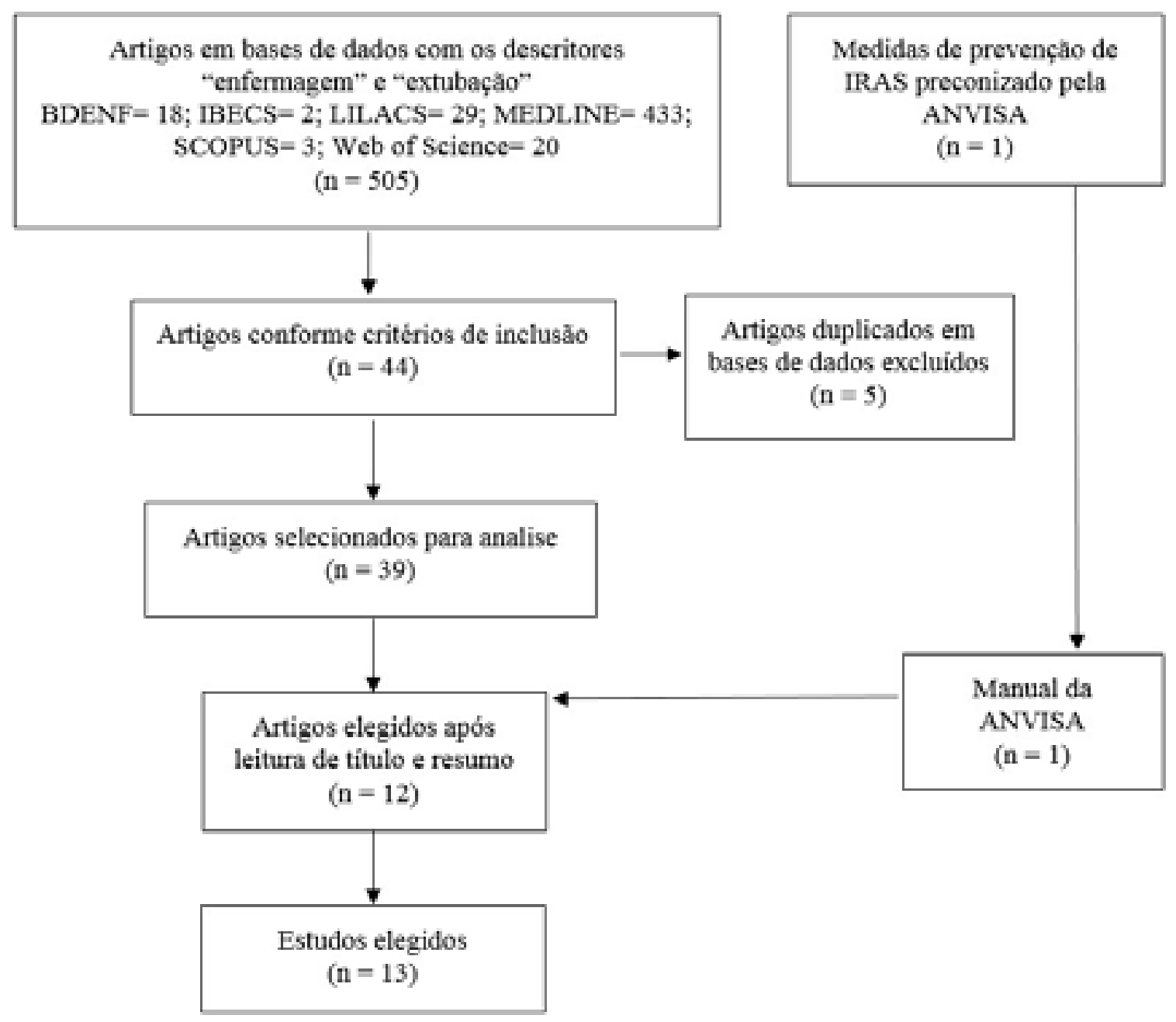

Figura 1 - Seleção de estudos científicos em bases de dados. Curitiba, Paraná, Brasil, 2018 
A busca resultou em 505 artigos dos quais 44 artigos foram selecionados por estarem de acordo com os critérios de inclusão. Excluídos cinco artigos duplicados que estavam em duas fontes ou mais, totalizando 39 artigos em bases de dados. Após leitura do título e/ou resumos destes artigos, 12 estavam relacionados com o objetivo deste estudo ou relacionados ao tema em questão.

O processo de análise dos dados foi realizado com a leitura de todos os trabalhos selecionados por dois revisores, a fim de determinar se estes apresentavam informações sobre o tema ou dados que estariam ligados a este estudo. Posteriormente foi realizado uma tabela com o resumo da metodologia, do resultado e da conclusão apresentados por estes estudos.

\section{RESULTADOS}

O Tabela 1 mostra os estudos encontrados que cumprem com todos os critérios de elegibilidade.

Tabela 1 - Estudos elegidos sobre cuidados de enfermagem no processo de extubação orotraqueal. Curitiba, Paraná, Brasil, 2018 (continua)

\section{IDENTIFICAÇÃO Autor/Ano/Título \\ METODOLOGIA}

Wang, Ma e Fang (2013)

Extubation with or without spontaneous breathing tria/(13)
Estudo prospectivo randomizado duplo cego observou pacientes da UTI geral de um hospital escola, em ventilação mecânica a serem extubados. Os pacientes foram separados em dois grupos durante o período de desmame de ventilação mecânica, sendo que um realizou teste de respiração espontânea e outro não.

Fonseca, Azzolin e Vieira (2014) Fatores associados ao tempo de ventilação mecânica nos pósoperatórios de cirurgia cardíaca $^{(14)}$

\section{Rodrigues e} colaboradores (2012) Ventilação mecânica: evidências para o cuidado de enfermagem ${ }^{(15)}$

Villa, Manara e Palese (2012)

Nurses' near-decisionmaking process of postoperative patients' cardiosurgical weaning and extubation in an Italian environment ${ }^{(16)}$
Estudo retrospectivo longitudinal analisou prontuários de pacientes submetidos a cirurgia cardíaca entre 2012 e 2013 na UTI de um hospital geral.

\section{Estudo transversal com} abordagem quantitativa aplicando questionários sobre ventilação mecânica para enfermeiros de 3 UTls de um hospital.
Estudo qualitativo etnográfico observou enfermeiros em seu trabalho e os entrevistou sobre o processo de extubação em pacientes de uma UTI Cardíaca.

\section{RESULTADOS E CONSIDERAÇÕES}

Durante a extubação dos pacientes em ventilação mecânica não houve diferença significativa entre os grupos. A prática de realizar o teste de respiração espontânea fazia parte do protocolo para desmame de paciente em ventilação mecânica da UTI desse hospital no qual a tomada de decisão é realizada pela colaboração mutua entre médicos e enfermeiros.

Alguns fatores foram associados com o aumento do tempo de permanência do TOT como: tempo de cirurgia, o uso de drogas para sedação, analgesia ou vasoconstrição.
A maioria dos enfermeiros respondeu que não participam do processo de extubação ou realizam aspiração traqueal.
Os enfermeiros assistenciais sugeriram realizar a extubação de acordo com os critérios de estado de consciência pela escala de Glasgow, temperatura corporal, estabilidade hemodinâmica, estabilidade dos parâmetros respiratórios (frequência respiratória - FR, pressão positiva expiratória - PEEP, fração de oxigênio inspirado - $\mathrm{FiO}^{2}$ ) e tempo decorrido pós-cirurgia, pois os fatores clínicos são importantes nesses processos. 
Silva, Nascimento e Salles (2014)

Pneumonia associada à ventilação mecânica: discursos de profissionais acerca da prevenção(17)
Estudo descritivo qualitativo entrevistou a equipe de enfermagem e os fisioterapeutas de uma UTI geral sobre conhecimentos acerca da prevenção de pneumonia associada à ventilação mecânica.
Preisig e colaboradores (2014)

Ventilação não invasiva após cirurgia cardiovascular: um Ensaio Clínico Randomizado ${ }^{(18)}$
Estudo com pacientes no pós-operatório imediato de cirurgia cardiovascular após a extubação. A extubação foi realizada de acordo com: nível de consciência, temperatura corporal, estabilidade hemodinâmica, sangramento ativo que necessite de reintervenção cirúrgica e tolerância ao teste de respiração espontânea.

\section{Jeon e colaboradores} (2016) Impact of delirium on weaning from mechanical ventilation in medical patients $^{(19)}$

Duan e colaboradores (2015)

Semiquantitative cough strength score for predicting reintubation after planned extubation ${ }^{(20)}$

Estudo prospectivo com analise dos registros dos pacientes em processo de desmame de ventilação mecânica em uma UTI e que foram diagnosticados com Delirium.

\section{Estudo experimental com} pacientes com TOT da UTI de um hospital universitário em processo de desmame, para testar a correlação entre a força de tosse e pico de fluxo de tosse. Realizado extubação desses pacientes pelo protocolo do Hospital em questão, com elevação de cabeceira de $30^{\circ}$ a $45^{\circ}$ graus durante $o$ processo, higiene das mãos dos profissionais envolvidos, higiene oral do paciente, verificação do nível de sedação pela escala de Ramsey e aspiração orotraqueal.

Jiang e colaboradores (2014)

Predicting weaning and extubation outcomes in long-term mechanically ventilated patients using the modified Burns Wean Assessment Program scores $^{(21)}$
Estudo de coorte retrospectivo com coleta dos dados clínicos de pacientes com diagnóstico de insuficiência respiratória em processo de desmame. Os pacientes em ventilação mecânica prolongada foram avaliados com protocolo Burns modificado, utilizado para predizer o momento apropriado para extubação.
A utilização de um protocolo ou rotina para o processo de desmame e extubação orotraqueal é eficiente e deve ser considerado para a predição desse processo em pacientes em ventilação mecânica prolongada. 
Sedwick e colaboradores (2012)

Using evidence-based practice to prevent ventilator-associated Pneumonia(22)
Estudo descritivo quali e quantitativo com aplicação um protocolo estruturado para expandir os cuidados já existentes com pacientes em ventilação mecânica, acrescentando ao protocolo já existente medidas como aplicação de cuidados bucais, higienização das mãos, alarmes de beira de leito, aspiração de vias aéreas, e outras ferramentas que apresentassem um feedback de conformidade a fim de diminuir os custos hospitalares com pneumonia associada à ventilação.

\section{Tanios e colaboradores} (2014)

Influence of

sedation estrategies on unplanned extubation in a mixed intensive care unit $^{(23)}$
Estudo retrospectivo avaliou casos de extubação não planejada dos pacientes em ventilação mecânica, associada à estratégia de sedação da UTI multidisciplinar de um hospital escola utilizando escala de agitação e sedação de Richmond (RASS).
Nassar Junior [tese doutorado] (2015) Impacto da sedação intermitente ou interrupção diária da sedação em pacientes sob ventilação mecânica(24)
Estudo prospectivo randomizado avaliou pacientes internados em UTI, sedados para ventilação mecânica e submetidos à interrupção dos sedativos divididos em dois grupos: interrupção intermitente ou diária.
O grupo interdisciplinar formado por enfermeiro, médico e fisioterapeuta implantou esse projeto e percebeu que houve um aumento da aderência dos profissionais às práticas do protocolo já existente (profilaxia para doenças vasculares, interrupção diária da sedação para monitorização de possível extubação e elevação da cabeceira do leito).
O número de extubações não planejadas foi diferente de acordo com a estratégia de sedação. A monitorização e/ou o planejamento de extubação devem ser parte do cuidado da equipe multidisciplinar a fim de limitar a ocorrência de agitações do paciente.
Não foi observada nenhuma diferença entre os dois grupos. Porém a interrupção diária da sedação se mostrou eficaz na redução do tempo de ventilação mecânica.

Para análise de intervenções preconizadas pelo Ministério da Saúde no Brasil, utilizouse o manual da ANVISA contendo medidas que visam a melhorar a segurança do paciente e a qualidade dos serviços de saúde e que contém alguns cuidados durante o desmame ventilatório e extubação endotraqueal(25). Os cuidados de enfermagem encontrados no documento são: higiene das mãos; avaliar possibilidade de extubação e interrupção da medicação sedativa diariamente; avaliação neurológica; avaliação da temperatura corporal; avaliação da estabilidade hemodinâmica; monitorização de parâmetros respiratórios; manter o paciente em jejum; realizar sondagem enteral em posição gástrica ou pilórica quando necessário; manter cabeceira do leito elevada de 30 a 45 graus; realizar higiene oral com clorexidina ou outro antisséptico bucal; aspiração da cavidade oral e de vias aéreas superiores; verificar pressão 0 do cuff para retirada do TOT; observar o momento da retirada do TOT; utilizar ventilação mecânica não invasiva após extubação; realizar umidificação de vias aéreas com inalação e nebulização.

De acordo com esses dados, podemos observar a formação de um conjunto de cuidados do processo de extubação orotraqueal, que podem ser realizados antes, durante ou após a extubação propriamente dita necessários na rotina das UTIs, como descrito na Tabela 2. 
Tabela 2 - Cuidados de enfermagem no processo de extubação orotraqueal. Curitiba, Paraná, Brasil, 2018

Cuidados de enfermagem

Avaliar e discutir com a equipe multiprofissional sobre o momento adequado para extubação

Observar a administração de medicação analgésica e sedativa

Se paciente cirúrgico, observar qual foi o tempo da cirurgia e o tempo pós-cirúrgico

Higienizar as mãos e enfatizar a higienização das mãos da equipe de enfermagem

Avaliar nível de consciência pela
escala de Glasgow e escala de
Ramsey ou escala de RASS

Avaliar temperatura corporal

Objetivo

Prever qual o melhor momento para extubação de acordo com os protocolos da instituição

Certificar a suspensão das medicações de sedação e analgesia

Evitar extubação precoce do paciente pós- cirúrgico

Momento da extubação

Antes Durante Depois

$X$

$\mathrm{X}$

$\mathrm{X}$

Prevenir infecções relacionadas

$\mathrm{X}$

à assistência de saúde pela

transmissão de microrganismos pelo

profissional de enfermagem

Observar grau de alerta

$x \quad x$
$x$
neurológicos

Identificar indicativos de infecções

$x \quad x$

respiratórias, tal como elevação da

temperatura corporal, que podem

dificultar a respiração espontânea

Avaliar estabilidade hemodinâmica

Verificar sinais fisiológicos (sinais

vitais, diurese, saturação de $\mathrm{O} 2$,

eletrocardiograma, pressão arterial

média e outros

Avaliar estabilidade respiratória com ausculta pulmonar, teste de respiração espontânea e de tosse e monitorar de parâmetros respiratórios (FR, $\mathrm{FiO}^{2}$ e $\mathrm{PEEP}$ )

Certificar que o paciente está apto

$x$ para respirar espontaneamente e verificar se há necessidade de realizar aspiração de vias aéreas

$x \quad x$

Manter o paciente em jejum

Realizar sondagem enteral em posição gástrica ou pilórica quando necessário

Manter cabeceira do leito elevada de
30 a 45 graus
30 a 45 graus

Realizar higiene oral com clorexidina ou outro antisséptico bucal

Aspirar cavidade oral e de vias aéreas superiores pelo TOT

Evitar bronco aspiração de resíduos estomacais

Manter a oferta de nutrientes caso

haja de dificuldade de deglutição

$x \quad x$

X

Evitar bronco aspiração e facilitar a troca de gases pulmonar

Evitar infecção de vias aéreas por resíduos bucais

Evitar infecção e retirar secreções de vias aéreas superiores que dificultam a troca de gases

Verificar pressão 0 do cuff para retirada do TOT

Auxiliar no momento da retirada do TOT
Facilitar a retirada do cuff e evitar traumas faríngeos e/ou laríngeos

A presença do enfermeiro pode diminuir complicações observando parâmetros respiratórios e realizando aspiração orotraqueal

Ofertar umidificação e O2

necessários para manter saturação de O2 mínima
Realizar umidificação de vias aéreas com inalação e nebulização (utilizar ventilação não invasiva) 
A utilização do TOT é um tratamento que permite a abertura e permeabilidade das vias aéreas, além de proporcionar cuidados específicos como o controle de parâmetros respiratórios por meio da ventilação mecânica. A complexidade desse tratamento envolve tanto a intubação quanto a extubação orotraqueal e ambas estão associadas a adversidades ${ }^{(26)}$.

Estudos mostram que a formação de protocolos e rotinas para o momento da extubação orotraqueal são de suma importância, pois tornam claros e objetivos os principais cuidados necessários para organizar a assistência de enfermagem e prevenir complicações ${ }^{(21-23)}$.

O conjunto dos estudos abordados nesta revisão norteia para os cuidados de enfermagem para a redução das IRAS, tais como higiene das mãos, higiene oral com Gluconato de Clorexidina $0,12 \%$ do paciente, aspiração orotraqueal e de vias aéreas superiores, posicionamento no leito com elevação de cabeceira, controle da pressão do cuff do TOT, evitar sedação desnecessária enquanto desmame ventilatório, prever o desmame ventilatório para o momento da extubação. Nota-se que há uma constante a respeito desses cuidados, para redução da microbiota local com potencial de gerar infecções e bronco aspiração( ${ }^{(3,22,26)}$.

As medidas essenciais para prevenção de IRAS contêm uma gama extensa de cuidados específicos, relacionados principalmente ao processo de desmame ventilatório. Visando aumentar a segurança do paciente no momento de retirada do tubo orotraqueal, essas intervenções são direcionadas tanto para equipe de enfermagem quanto para outros profissionais de saúde ${ }^{(26)}$.

Verificou-se a importância de aplicar avaliação neurológica e do nível de consciência do paciente, uma vez que o paciente pode apresentar dificuldade para manter a respiração espontânea sem o uso do TOT pelo efeito prolongado das medicações sedativas no momento da extubação ${ }^{(19,24)}$.

Os critérios para realização da extubação devem incluir avaliação neurológica, temperatura corporal, estabilidade hemodinâmica e respiratória com teste de respiração espontânea para saber quais são as condições fisiopatológicas do paciente ${ }^{(16,18)}$. A execução destes testes visa reduzir os riscos de falha da extubação, como exemplo o delirium, que é um estado de confusão mental aguda, e que está diretamente associado à dificuldade de desmame ventilatório e necessidade de reintubação do paciente em UTI(19).

Um estudo mostra desacordo no item sobre a necessidade de teste de respiração espontânea, devendo a equipe de enfermagem e os médicos decidirem o melhor momento para extubação de maneira mais subjetiva(13). Porém, outro sugere que um teste de tosse no processo de extubação é indispensável para a extubação(20).

Quanto à restrição de sedação e analgesia, diversos autores concordam que deve ser realizada uma avaliação diária da medicação de sedação e analgesia a fim de avaliar a possibilidade de extubação(13-14,19-20,22-24).

A oxigenação imediata por névoa inalatória que garante um suporte de oxigênio mínimo, a manutenção do jejum associado à verificação da sonda nasogástrica ou nasoenteral também são indicadas para prevenir bronco aspiração de conteúdo gástrico ${ }^{(17)}$.

Além dos cuidados diretos na assistência de enfermagem, os autores também explanam sobre a interdisciplinaridade da equipe multiprofissional no momento da extubação, no qual deve haver coesão construída por discussões interprofissionais relacionadas principalmente aos enfermeiros e outros profissionais de saúde ${ }^{(13,15,17,22)}$.

Um dos estudos encontrou uma diminuição da participação do enfermeiro nessas discussões, sugerindo que poucos são os enfermeiros que realizam cuidados associados a 
esse processo, apesar de terem competência para avaliar parâmetros ventilatórios e opinar nas discussões da equipe multiprofissional, pela alta demanda desses setores ${ }^{(15)}$.

Por essa razão, sustenta-se o primeiro ponto da Tabela 2, sobre a garantia de planejamento da equipe multiprofissional para definir o momento mais adequado para a extubação orotraqueal. Esse ponto define o início de uma rotina de cuidados de enfermagem durante o processo de extubação orotraqueal, a fim de garantir a segurança do paciente e permitir a qualidade dos serviços da equipe de enfermagem.

Observam-se limitações no presente estudo. A primeira delas é a heterogeneidade dos estudos selecionados, uma vez que o objeto de cada um é significativamente distante entre si. E para atingir o objetivo da pesquisa, houve necessidade de buscar na literatura pesquisada os termos que mais se assemelham ao processo de extubação orotraqueal para obter seus cuidados de enfermagem. Outra limitação foi a dificuldade de análise dos dados para extrair os cuidados de enfermagem específicos para o processo da extubação orotraqueal.

\section{CONCLUSÃO}

O envolvimento do enfermeiro e sua equipe no processo de extubação contribui para assegurar a integridade do paciente, prevenir complicações, reduzir o tempo de internação e os custos associados a UTI. Portanto, criar protocolos para que os cuidados de enfermagem estejam embasados nas melhores práticas, tornam claras as atribuições e responsabilidades do enfermeiro. Ao sistematizar essas práticas, o profissional se torna indispensável no planejamento da assistência de qualidade.

Sendo assim, o enfermeiro e a equipe de enfermagem devem buscar manter-se atualizados e capacitados para realizar com excelência suas ações. Também se espera com este estudo explanar sobre a importância da atuação do enfermeiro nos cuidados ao paciente crítico.

Sugerem-se outros estudos para compreender a percepção do enfermeiro frente às atividades relacionadas ao processo multiprofissional de extubação orotraqueal no momento de desmame ventilatório e extubação orotraqueal, e verificar o impacto destes cuidados na redução de IRAS.

\section{REFERÊNCIAS}

1. Barbosa TP, Oliveira GA de, Lopes MN, Poletti NA, Beccaria LM. Práticas assistenciais para segurança do paciente em unidade de terapia intensiva. Acta Paul Enferm. [Internet]. 2014 [acesso em 08 jan 2018]; 27(3). Disponível em: http://dx.doi.org/10.1590/1982-0194201400041.

2. Oliveira HM de, Silva CPR, Lacerda RA. Policies for control and prevention of infections related to healthcare assistance in Brazil: a conceptual analysis. Rev Esc Enferm USP. [Internet]. 2016 [acesso em 08 jan 2018]; 50(3). Disponível em: http://dx.doi.org/10.1590/S0080-623420160000400018.

3. Leal GA, Ribeiro JB, Santos JJ dos, Cavalcante AB. Cuidados de enfermagem para prevenção de pneumonia associada a ventilação mecânica em Unidades de Terapia Intensiva: uma revisão literária. Cad. Ciênc. Biol. Saúde [Internet]. 2017 [acesso em 09 nov 2017]; 4(1). Disponível em: https://periodicos.set. edu.br/index.php/cadernobiologicas/article/view/3657.

4. Duarte SCM, Stipp MAC, Mesquita MGR, Silva MM da. O cuidado de enfermagem no pós-operatório de cirurgia cardíaca: um estudo de caso. Esc. Anna Nery. [Internet]. 2012 [acesso em 18 set 2017]; 16(4). Disponível em: http://dx.doi.org/10.1590/S1414-81452012000400003.

5. Danckers M, Grosu H, Jean R, Cruz RB, Fidellaga A, Han Q, et al. Nurse-driven, protocol-directed 
weaning from mechanical ventilation improves clinical outcomes and is well accepted by intensive care unit physicians. J Crit Care [Internet]. 2013 [acesso em 21 ago 2017]; 28(4) Disponível em: http://dx.doi. org/10.1016/j.jcrc.2012.10.012.

6. Franco JB, Jales SMCP, Zambon CE, Fujarra FJC, Ortegosa MV, Guardieiro PFR, et al. Higiene bucal para pacientes intubados sob ventilação mecânica assistida na unidade de terapia intensiva: proposta de protocolo. Arq Med Hosp Fac Cienc Med Santa Casa São Paulo [Internet]. 2014 [acesso em 08 jan 2018]; 59(3). Disponível em: http://www.fcmsantacasasp.edu.br/images/Arquivos medicos/2014/59 3/05-AO74. pdf.

7. Ward D, Fulbrook P. Nursing strategies for effective weaning of the critically ill mechanically ventilated patient. Crit Care Nurs Clin North Am. [Internet]. 2016. [acesso em 08 jan 2018]; 28(4). Disponível em: http://dx.doi.org/10.1016/j.cnc.2016.07.008.

8. Rocha AEF, Rocha FAA, Mourão Neto JJ, Gomes FMA, Cisne MSV. Cuidado de enfermagem ao paciente ventilado artificialmente: uma revisão integrativa. Essentia. [Internet]. 2017 [acesso em 09 nov 2017]; 18(1). Disponível em: http://www.uvanet.br/essentia/index.php/revistaessentia/article/view/35.

9. Shimizu JM, Manzano RM, Quitério RJ Alegria VTC, Junqueira TT, et al. Determinant factors for mortality of patients receiving mechanical ventilation and effects of a protocol muscle training in weaning. Man. Ther., Posturology Rehabil. J. [Internet]. 2014 [acesso em 19 jun 2017]; (12). Disponível em: http:// dx.doi.org/10.17784/mtprehabjournal.2014.12.180.

10. Melo EM, Teixeira CS, Oliveira RT de, Almeida DT de, Veras JEGLF, Frota NM, Studart RMB. Cuidados de enfermagem ao utente sob ventilação mecânica internado em unidade de terapia intensiva. Rev. Enf. Ref. [Internet]. 2014 [acesso em 19 jun 2017]; 4(1). Disponível em: http://www.scielo.mec.pt/pdf/ref/ vserlVn1/serlVn1a07.pdf.

11. Ercole FF, Melo LS de, Alcoforado CLGC. Revisão integrativa versus revisão sistemática. REME - Rev Min Enferm. [Internet]. 2014 [acesso em 05 jun 2017]; 18(1). Disponível em: http://dx.doi. org/10.5935/1415-2762.20140001.

12. Ganong LH. Integrative reviews of nursing research. Res Nurs Health. [Internet]. 1987 [acesso em 05 jun 2017]; 10(1). Disponível em: http://dx.doi.org/10.1002/nur.4770100103.

13. Wang J, Ma Y, Fang Q. Extubation With or Without Spontaneous Breathing Trial. Crit Care Nurse. [Internet]. 2013 [acesso em 29 jan 2018]; 33(6). Disponível em: http://dx.doi.org/10.4037/ccn2013580.

14. Fonseca L, Vieira FN, Azzolin KO. Factors associated to the length of time on mechanical ventilation in the postoperative period of cardiac surgery. Rev. Gaúcha Enferm. [Internet]. 2014 [acesso em 29 jan 2018]; 35(2). Disponível em: http://dx.doi.org/10.1590/1983-1447.2014.02.44697.

15. Rodrigues YCSJ, Studart RMB, Andrade IRC, Citó MCO, Melo EM, Barbosa IV. Ventilação mecânica: evidências para o cuidado de enfermagem. Esc. Anna Nery. [Internet]. 2012 [acesso em 29 jan 2018]; 16(4). Disponível em: http://dx.doi.org/10.1590/S1414-81452012000400021.

16. Villa G, Manara D, Palese A. Nurses' near decision making process of postoperative patients' cardiosurgical weaning and extubation in an Italian environment. Intensive Crit Care Nurs. [Internet]. 2012 [acesso em 29 jan 2018]; 28(1). Disponível em: http://dx.doi.org/10.1016/j.iccn.2011.10.007.

17. Silva SG da, Nascimento ERP do, Salles RK de. Ventilator-associated pneumonia: discourse of professionals about prevention. Esc Anna Nery. [Internet]. 2014 [acesso em 29 jan 2018]; 18(2). Disponível em: http://dx.doi.org/10.5935/1414-8145.20140042.

18. Preisig A, Lagni VB, Almeida VL de, Vieira FN, Lucio EA, Santos LJ dos, et al. Ventilação não Invasiva após Cirurgia Cardiovascular: um Ensaio Clínico Randomizado. Rev Bras Cardiol. [Internet]. 2014 [acesso em 29 jan 2018]; 27(1). Disponível em: http://www.onlineijcs.org/sumario/27/pdf/v27n1a07.pdf.

19. Jeon $\mathrm{K}$, Jeong $\mathrm{BH}$, Ko MG, Nam J, Yoo H, Chung CR, et al. Impact of delirium on weaning from mechanical ventilation in medical patients. Respirology [Internet]. 2016 [acesso em 29 jan 2018]; 21(2). Disponível em: http://dx.doi.org/10.1111/resp.12673. 
20. Duan J, Zhou L, Xiao M, Liu J, Yang X. Semiquantitative Cough Strength Score for Predicting Reintubation After Planned extubation. Am J Crit Care. [Internet]. 2015 [acesso em 29 jan 2018]; 24(6). Disponível em: http://dx.doi.org/10.4037/ajcc2015172.

21. Jiang JR, Yen SY, Chien JY, Liu HC, Wu YL, Chen CH. Predicting weaning and extubation outcomes in long-term mechanically ventilated patients using the modified Burns Wean Assessment Program scores. Respirology [Internet]. 2014 [acesso em 29 jan 2018]; 19(4). Disponível em: http://dx.doi.org/10.1111/ resp. 12266.

22. Sedwick MB, Lance-Smith M, Reeder SJ, Nardi J. Using evidence-based practice to prevent ventilatorassociated pneumonia. Crit Care Nurse. [Internet]. 2012 [acesso em 29 jan 2018]; 32(4). Disponível em: http://dx.doi.org/10.4037/ccn2012964.

23. Tanios M, Epstein S, Grzeskowiak M, Nguyen HP, Park H, Leo J. Influence of sedation estrategies on unplanned extubation in a mixed intensive care unit. Am J Crit Care [Internet]. 2014 [acesso em 29 jan 2018]; 23(4). Disponível em: http://dx.doi.org/10.4037/ajcc20144446.

24. Nassar Junior AP. Impacto da sedação intermitente e ou interrupção diária da sedação em pacientes sob ventilação mecânica [tese]. São Paulo (SP): Faculdade de Medicina Universidade de São Paulo; 2015.

25. Agência Nacional de Vigilância Sanitária (ANVISA). Série Segurança do paciente e qualidade em serviços de saúde. Medidas de Prevenção de Infecção Relacionada à Assistência à Saúde. Brasília: ANVISA, 2017.

26. Jacobs PE, Grabinsky A. Advances in prehospital airway management. International Journal of Critical Illness and Injury. Int J Crit Illn Inj Sci. [Internet]. 2014 [acesso em 15 fev 2018]; 4(1). Disponível em: http:// dx.doi.org/10.4103/2229-5151.128014.

Recebido: 27/02/2018

Finalizado: 13/03/2019

Autor Correspondente:

Tessy Nnonyelum Miozzo Ezeagu

Complexo do Hospital de Clínicas do Paraná

R. General Carneiro, 181 - 80060-900 - Curitiba, PR, Brasil

E-mail: tmiozeagu@yahoo.com.br

Contribuição dos autores:

Contribuições substanciais para a concepção ou desenho do estudo; ou a aquisição, análise ou interpretação de dados do estudo - TNME

Aprovação da versão final do estudo a ser publicado - ACGR 